\title{
Bloodstream infections in adult patients with malignancy, epidemiology, microbiology, and risk factors associated with mortality and multi-drug resistance
}

Ali Amanati ${ }^{1}$, Sarvin Sajedianfard², Somayeh Khajeh², Shabnam Ghasempour², Salma Mehrangiz², Samane Nematolahi ${ }^{2^{* t}}$ and Zahra Shahhosein ${ }^{2^{*}+}$

\begin{abstract}
Background: This study aimed to investigate the epidemiology, microbiology, and risk factors associated with mortality and multi-drug resistance bacterial bloodstream infections (BSIs) among adult cancer patients in Shiraz, Iran. We also report a four-year trend of antimicrobial resistance patterns of BSIs.
\end{abstract}

Methods: We conducted a retrospective study at a referral oncology hospital from July 2015 to August 2019, which included all adults with confirmed BSI.

Results: 2393 blood cultures tested during the four-year study period; 414 positive cultures were included. The mean age of our patients was $47.57 \pm 17.46$ years old. Central Line-Associated BSI (CLABSI) was more common in solid tumors than patients with hematological malignancies. Gram-negative (GN) bacteria were more detected $(63.3 \%, 262)$ than gram-positive bacteria $(36.7 \%, 152)$. Escherichia coli was the most common gram-negative organism (123/262, 47\%), followed by Pseudomonas spp. (82/262, 31\%) and Klebsiella pneumoniae (38/262, 14.5\%). Coagulase-negative staphylococci (CONS) was the most frequently isolated pathogen among gram-positive bacteria (83/152, 54.6\%). Acinetobacter spp., Pseudomonas spp., E. coli, and K. pneumoniae were the most common ExtendedSpectrum Beta-Lactamase (ESBL) producers (100, 96.2, 66.7\%, and 60.7, respectively). Acinetobacter spp.,

Pseudomonas spp., Enterobacter spp., E. coli, and K. pneumoniae were the most common carbapenem-resistant (CR) isolates (77.8, 70.7, 33.3, 24.4, and 13.2\%, respectively). Out of 257 Enterobacterales and non-fermenter gram-negative BSIs, 39.3\% (101/257) were carbapenem-resistant. Although the incidence of multi-drug resistance (MDR) gramnegative BSI increased annually during 2015-2018, the mortality rate of gram-negative BSI remains unchanged at about 20\% ( $p$-value $=0.55)$; however, the mortality rate was significantly greater $(35.4 \%)$ in those with resistant gram-positive BSI ( $p$-value $=0.001)$. The overall mortality rate was $21.5 \%$. Early (7-day mortality) and late mortality rate (30-day mortality) were 10 and 3.4\%, respectively.

\footnotetext{
*Correspondence: nshahhosein@yahoo.com;

samane.nematolahi@yahoo.com

†'Samane Nematolahi and Zahra Shahhosein contributed equally to this work.

${ }^{2}$ Shiraz University of Medical Sciences, Shiraz 7193711351, Iran

Full list of author information is available at the end of the article
} 
Conclusions: The emergence of MDR gram-negative BSI is a significant healthcare problem in oncology centers. The high proportion of the most frequently isolated pathogens were CR and ESBL-producing Enterobacterales and Pseudomonas spp. We have few effective choices against MDRGN BSI, especially in high-risk cancer patients, which necessitate newer treatment options.

Keywords: Bloodstream infection, Carbapenem-resistant isolates, Extended-Spectrum Beta-lactamase producing pathogens, Multidrug-resistant gram-negative infection, Mortality, Cancer

\section{Background}

Bacterial bloodstream infection (BSI) is one of the most common complications of chemotherapy-induced neutropenia in patients with hematologic malignancies and solid organ tumors [1-3], which is associated with high mortality and morbidity [4-8]. Bacterial BSIs account for the etiologic cause of approximately 20 to $30 \%$ of all febrile neutropenic episodes in adult patients with malignancy $[9,10]$. While proper diagnosis and treatment are essential to decrease BSI-associated complications, inappropriate empiric antimicrobial therapy increases mortality [11]. BSI's reported crude mortality rates to reach as high as 34 to $50 \%$, especially in MDR gramnegative BSI $[9,12]$. The antimicrobial stewardship program (ASP) helps to reduce the overuse of antibiotics and control the increased antimicrobial resistance. Surveillance of antimicrobial resistance is one of ASP's critical aspects and guides clinicians for appropriate empiric antimicrobial therapy $[13,14]$.

This study aimed to determine the current epidemiology of bacterial BSI and its changes during the different study years in a large cohort of patients with solid organ and hematological malignancy. We also assessed BSI attributed mortality risk factors and MDR gramnegative BSI predictors.

\section{Methods}

\section{Setting and data collection}

This study performed at Amir oncology hospital, an educational 100-bed inpatient center. The adult units consist of four inpatient wards and an autologous hematopoietic stem cell transplantation ward. Since 2015 our institution has carried out a blood culture surveillance program using an automated blood culture system (BD BACTEC ${ }^{\mathrm{mm}}$ ). Hospital Information System (HIS) and microbiology department records used for data collection. Patients followed up 30 days after BSI by infectious disease specialist consultant (AA) and chief infection control unit staff (MS).

\section{Study population and design}

In this retrospective single-center study, we analyzed all consecutive episodes of BSI occurring in adult patients with hematological malignancies and solid organ tumors from July 2015 to August 2019. Each patient $\geq 18$ years of age with positive blood culture was considered to BSI when he/she had clinical signs and symptoms of bacteremia. Patients with a fungal infection, contaminant result, or a mix of more than two organisms, excluded from this study. Finally, 414 patients recruited, and data analyzed for age, gender, underlying diseases, presence of central venous catheters, leucocyte count, neutropenia, etiologic microorganisms, susceptibility testing, and outcome.

\section{Definitions}

Patient with a recognized bacterial pathogen, which not included on the commensal list, identified from one or more blood specimens obtained by culture and at least one of the following signs or symptoms: fever $\left(>38^{\circ} \mathrm{C}\right)$, chills, or hypotension and not be related to an infection at another site considered as true BSI [15].

Febrile neutropenia defined as temperature $>38.5^{\circ} \mathrm{C}$ or two consecutive temperature $>38^{\circ} \mathrm{C}$ for $2 \mathrm{~h}$ and an absolute neutrophil count $<0.5 \times 10^{9}$ cell/L or expected to fall below $<0.5 \times 10^{9}$ cell/L. Imipenem, meropenem, cefepime, piperacillin-tazobactam, and colistin are available on our hospital formulary. We use piperacillintazobactam and carbapenem as the first-line agents for patients with febrile neutropenia.

Methicillin-resistant coagulase-negative staphylococci (MRCoNS) defined as cefoxitin-resistant strains. Enterobacteriaceae family, P. aeruginosa, and A. baumannii isolates resistant to ceftazidime or cefotaxime are considered extended-spectrum beta-lactamase (ESBL) producers [16]. Phenotypic confirmation of ESBL production carried out by using the double-disk synergy test [17]. Carbapenemresistant Enterobacterales (CREs), carbapenem-resistant Pseudomonas spp., and carbapenem-resistant Acinetobacter spp. isolates defined as Enterobacterales that test intermediate or resistant to one or more carbapenems using the CLSI current breakpoints. However, not all isolates tested against all carbapenems [16]. MDR defined as the strain non-susceptible to at least one agent in $\geq 3$ classes of antibiotics, including carbapenems, combinations of betalactams plus beta-lactamase inhibitors, cephalosporins, aminoglycosides, and fluoroquinolones [18].

\section{Microbiological methods}

Blood cultures obtained by physicians based on clinical suspicion of bacteremia. BACTEC ${ }^{\mathrm{Tm}}$ FX Automated 
Blood Culture Systems used for detection of BSI. Timeto-Detection (TTD) defined as the time between the placement of each blood culture bottle in the incubation cabinet and the detection of growth. Based on the result of the gram-stain, the subculture routinely was performed, and differentiation tests such as catalase, oxidase, coagulase, bacitracin, optochin, and CAMP test were applied. In our center, the susceptibility testing is done based on the disc diffusion method (Kirby-Bauer method) according to the Clinical and Laboratory Standards Institute (CLSI) guidelines [17] using commercial antibiotic discs (MAST Group Ltd.; UK).

\section{Statistical analysis}

In this study, we used a univariate logistic regression model to examine the critical factors that may be influencing the patient's survival status and possible predictors of MDR gram-negative BSI. All variables in the univariate analysis $(P \leq 0.25)$ and variables with clinical significance entered into a multivariable model. The independent variables of sex, age, malignancy, ESR, CRP, leukopenia, neutropenia, febrile neutropenia, year, and Enterobacterales separately entered a logistic regression model. We reported odds ratio values and also the confidence interval of the odds ratio for each variable. A $p$ value $<0.05$ was considered statistically significant. The analysis was done by SPSS version 25 (IBM Corp., Armonk, NY, USA).

\section{Results}

\section{Demographics and epidemiology}

In this study, four-hundred, fourteen positive blood cultures were analyzed (Fig. 1). Two hundred twenty-three patients were male (53.9\%). The mean age was $47.57 \pm$ 17.46 years old. Hematologic malignancies (212, 51.4\%), solid tumors $(167,40.3 \%)$, and non-malignant disorders (20, 4.8\%), including aplastic anemia, were the most common underlying diseases, respectively.

\section{Clinical and laboratory features}

The mean of white blood cell (WBC), erythrocyte sedimentation rate (ESR), and C-reactive protein (CRP) were $(6.94 \pm 11.28) \quad X \quad 10^{9} / \mathrm{L}, \quad(71.68 \pm 37.26) \mathrm{mm} / \mathrm{hour}$, $(82.59 \pm 41.44) \mathrm{mg} / \mathrm{dl}$, respectively. ESR and CRP were not statistically different between two malignancy types $(P=0.6646$; $95 \%$ CI: -7.07 to 11.07 and $P=0.0663,95 \%$ CI: -19.64 to 0.64 , respectively). Neutropenia was more prominent in those with hematologic malignancies (117, $69.6 \%)$ compare with patients with solid organ tumors $(38,22.6 \%) ; P<0.001$. Febrile neutropenia also was significantly higher in those with hematologic malignancies (83, 74.8\%; $\mathrm{P}<0.001$ ). Overall, $63.5 \%$ of positive culture was primary BSI, and $36.5 \%$ was CLABSI. CLABSI was more common in those with solid tumors; the difference

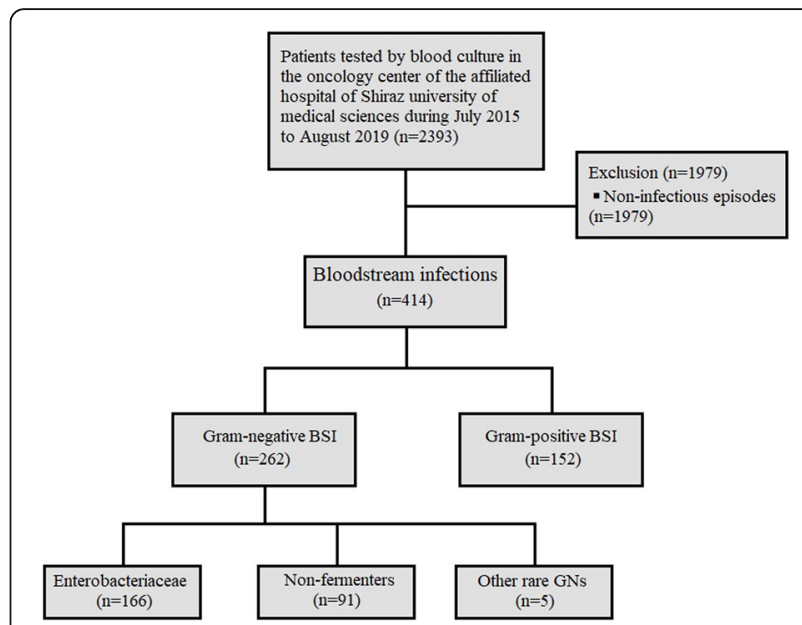

Fig. 1 Flow chart of study population screened and enrolled associated with bloodstream infections caused by gram-negative and gram-positive bacteria in patients treated at the Amir oncology hospital between July 2015 to August 2019

was not significant $p=0.165$. The most reported TTD was less than $12 \mathrm{~h}(188 / 414,50 \%)$, while $30.3 \%$ detected during $12-24 \mathrm{~h}, 11.7 \%$ between 24 and $36 \mathrm{~h}$, and only $8 \%$ diagnosed in more than $36 \mathrm{~h}$ by BACTEC ${ }^{\mathrm{mm}}$ Systems. The rate of admission, BSI, febrile neutropenia, and mortality during the study years are shown in Fig. 2 . The summary characteristics of the patients with solid tumors and hematological malignancies could be found in Table S1 in the supplement file.

\section{Microbiology}

$262(63.3 \%)$ and 152 (36.7\%) gram-negative and grampositive pathogens organisms isolated from blood cultures. Escherichia coli was the most common gram-negative organism $(123 / 262,47 \%)$, followed by Pseudomonas spp. $(82 / 262,31 \%)$ and $K$. pneumoniae (38/262, 14.5\%). Coagulase-negative staphylococci (CoNS) was the most frequently isolated pathogen among gram-positive bacteria (83/152, 54.6\%). The incidence of gram-positive and gram-negative bacteria isolated from blood culture is shown in Fig. 3. Acinetobacter spp., Pseudomonas spp., E. coli, and $K$. pneumoniae were the most common ESBL producers (100, 96.2, 66.7\%, and 60.7, respectively).

Acinetobacter spp., Pseudomonas spp., Enterobacter spp., E. coli, and K. pneumoniae were the most common carbapenem-resistant isolates $(77.8,70.7,33.3,24.4$, and $13.2 \%$, respectively).

Out of 257 Enterobacterales and non-fermenter gramnegative BSIs, $39.3 \% \quad(101 / 257)$ were carbapenemresistant. The incidence of CRE and carbapenemresistant non-fermenter BSIs increased annually between 2015 and $2018(p<0.001)$. 


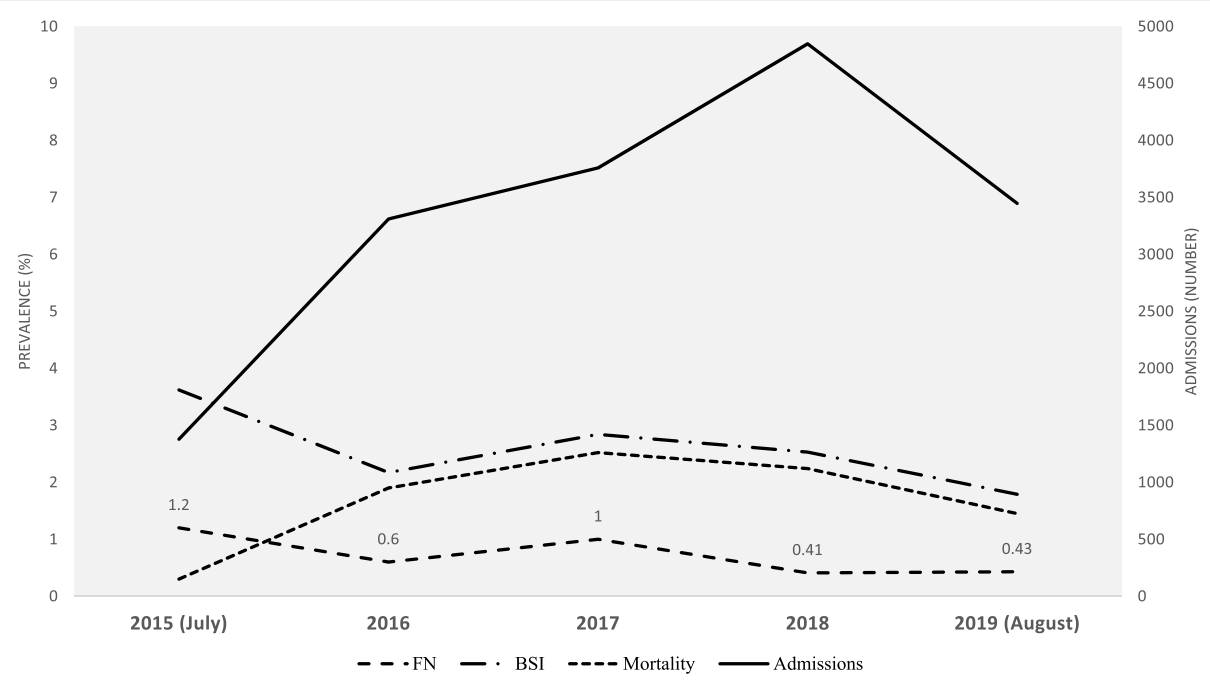

Fig. 2 Incidence of febrile neutropenia, bloodstream infections, mortality rate/10000 cases, and hospitalized adults with cancer during $2015-2019$. FN: febrile neutropenia episodes; BSI: bloodstream infection

The frequency of isolated organisms, MRCoNS, ESBL, and CRGN associated BSI, is shown in Fig. 4. As shown, there are emerging ESBL and CRGN BSIs during different study years.

Antibiotic susceptibility results for the most common isolated bacteria are shown in Figs. 5 and 6. All E. coli were sensitive to polymyxin b. The sensitivity of $E$. coli for meropenem, colistin, amikacin, and imipenem were 92, 82.79, and 78\%, respectively. All E. coli was resistant to piperacillin-tazobactam. More than $90 \%$ of Pseudomonas spp. were sensitive to ciprofloxacin, amikacin, gentamicin, and polymyxin-b, while $97 \%$ were chloramphenicol- resistant. All K. pneumoniae was sensitive to polymyxin-b, and $91 \%$ were susceptible to amikacin. More than $80 \%$ of $K$. pneumoniae was sensitive to meropenem, imipenem, and colistin. Trimethoprim/ sulfamethoxazole and ampicillin-sulbactam found less sensitive agents. Most coagulase-negative staphylococci (94\%) were susceptible to linezolid, and more than $80 \%$ of them were susceptible to teicoplanin, rifampicin, chloramphenicol, and vancomycin.

Among the Enterobacteriaceae family, 91 (64.1\%) isolates were ESBL-producer, and 36 (28.8\%) were CRE. $\mathrm{CR}$ detected in $71.6 \% P$. aeruginosa and $87.5 \%$ of Acinetobacter spp.

Overall, in those with gram-negative BSI, 49.3\% found to be non-susceptible to at least one agent in $\geq 3$ classes of antibiotics, including carbapenems (imipenem or meropenem), combinations of beta-lactams plus beta-lactamase inhibitors (piperacillin-tazobactam or ampicillin-sulbactam),

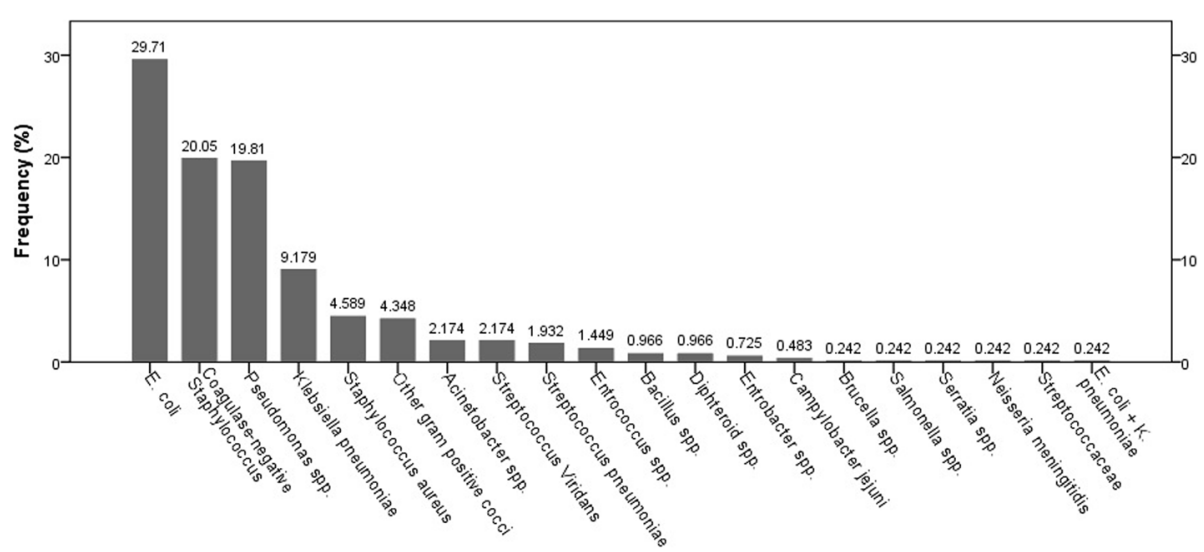

Fig. 3 The percentage frequency distribution of different gram-negative and gram-positive bacteria isolated from blood cultures 
\% CRGN $\otimes E S B L \square M R C O N S$

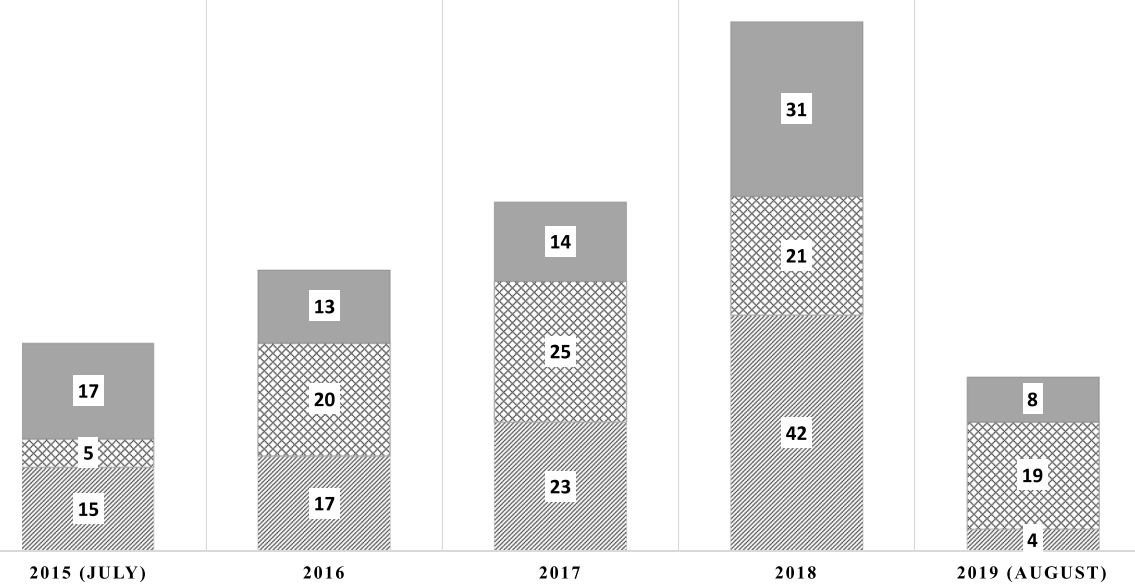

Fig. 4 The annual frequency of ESBL and CRE-associated BSI, in addition to MRCoNS, associated BSI (labels represent case numbers). CRGN includes carbapenem resistance Enterobacterales and non-fermenter spp.
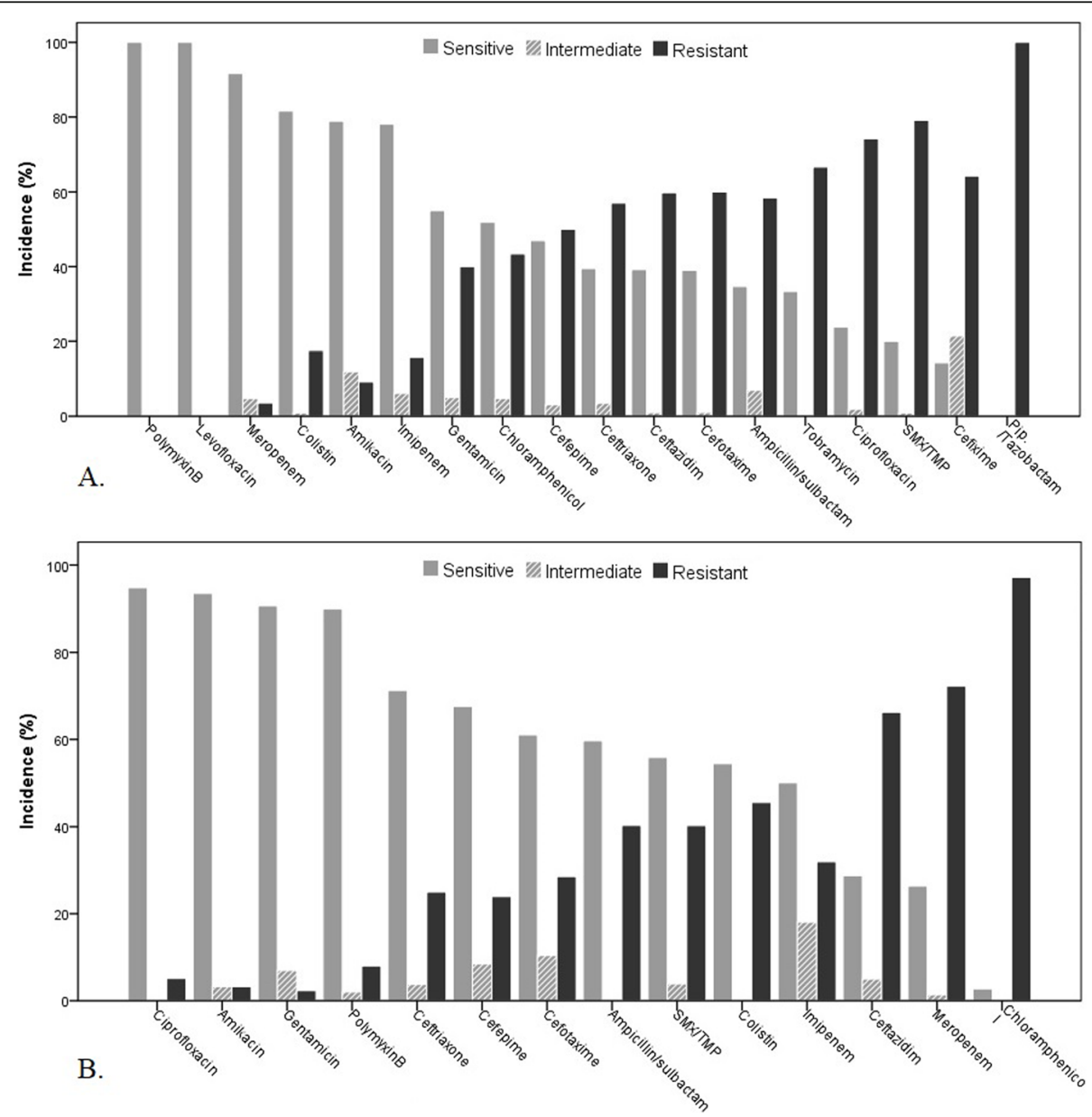

Fig. 5 Antimicrobial susceptibility results of 123 E. coli (A) and 81 Pseudomonas spp. (B) isolates recovered from blood cultures during 2015-2019 

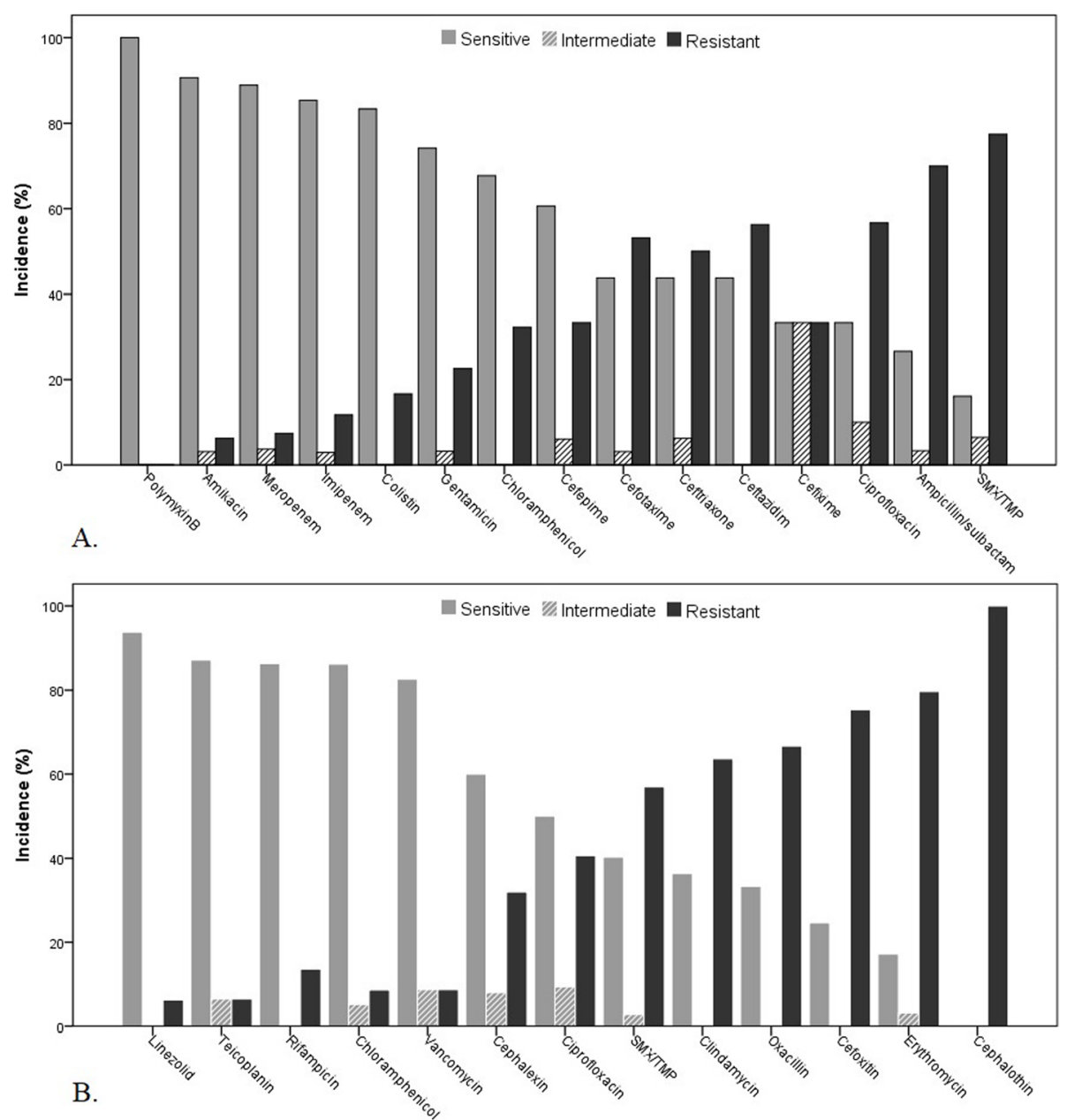

Fig. 6 Antimicrobial susceptibility results of 38 K. pneumonia (A) and of 80 coagulase-negative staphylococci (CoNS) isolates (B) recovered from blood cultures during 2015-2019

cephalosporins (3rd or 4th generation cephalosporins), aminoglycosides (amikacin or gentamicin), or fluoroquinolones (ciprofloxacin or levofloxacin). Although the MDR gramnegative (MDRGN) BSI cases increased from July 2015 to 2018 , the difference was not significant $(p=0.588)$. Table 1 represents the incidence of MRCoNS, ESBL producing organisms, CRE and Acinetobacter spp. and Pseudomonas spp. in different study years.

Among tested ESBL-producer gram-negative isolates, susceptibility to polymyxin-b, amikacin, colistin, imipenem, and meropenem, were $95,87,70,65.4$, and 54.5\%, respectively. Polymyxin-b, amikacin, and colistin found the most active agent against CRE clinical isolates (87.5, 75 , and $43.2 \%$, respectively).

Sixty cases detected as MRCoNS (76.9\%). Among MRCoNS, 94\% were susceptible to linezolid, and more than $80 \%$ were susceptible to teicoplanin, chloramphenicol, and rifampin, while vancomycin was less sensitive (74\%). All of the isolated enterococci were VRE.
Clinical influence of drug-resistance BSI and predictors of mortality

Based on the obtained results, third-generation cephalosporin-resistant $E$. Coli and P. aeruginosa, carbapenem-resistant $P$. aeruginosa, and polymyxinresistant $K$. pneumoniae BSIs associated with increased mortality; however, the difference was not statistically significant ( $p$-value: $0.719,>0.999,0.521$, and 0.467; respectively).

Based on results of univariate logistic regression analysis of variables investigated for survival in cancer patients with BSI, none of them associated with a greater risk of mortality (Table 2). Our results revealed that the odds of mortality decreased annually during 2015-2018 (Table 2).

Besides, univariate logistic regression analysis showed that only WBC count $<4000 / \mu \mathrm{l}$ (OR: 1.753; 1.159$2.651)$ and non-fermenter gram-negative BSI (OR: 3.120; 1.481-6.575) were associated with greater risk for MDR 
Table 1 MDRGN, ESBL, and CRGN associated BSIs frequency among most common gram-negative bacteria as well as MRSA and MRCONS among gram-positive associated BSIs during 2015-2019

\begin{tabular}{|c|c|c|c|c|c|c|c|}
\hline & & $\begin{array}{l}2015 \\
\text { n (\%) }\end{array}$ & $\begin{array}{l}2016 \\
\text { n (\%) }\end{array}$ & $\begin{array}{l}2017 \\
\text { n (\%) }\end{array}$ & $\begin{array}{l}2018 \\
\text { n (\%) }\end{array}$ & $\begin{array}{l}2019 \\
\text { n (\%) }\end{array}$ & p-value \\
\hline \multirow[t]{5}{*}{ MDR } & & $20(40)$ & $36(50)$ & $53(49.5)$ & $66(53.75)$ & $29(46.8)$ & 0.588 \\
\hline & E. coli & $12(60)$ & $22(61.1)$ & $26(49.1)$ & $13(19.7)$ & $18(62.1)$ & \\
\hline & K. pneumoniae & $3(15)$ & $3(8.3)$ & $4(7.5)$ & $9(13.6)$ & $5(17.2)$ & \\
\hline & Acinetobacter spp. & $2(10)$ & $3(8.3)$ & $2(3.8)$ & $0(0)$ & $0(0)$ & \\
\hline & Pseudomonas spp. & $1(5)$ & $6(16.7)$ & $18(34)$ & $43(65.2)$ & $6(20.7)$ & \\
\hline \multirow[t]{5}{*}{ ESBL } & & $5(15.2)$ & $31(46.3) *$ & $43(43) *$ & $66(55.5)$ & $28(45.9)$ & 0.002 \\
\hline & E. coli & $5(100)$ & $18(75)$ & $20(60.6)$ & $14(82.4)$ & $13(50)$ & \\
\hline & K. pneumoniae & $0(0)$ & $3(15)$ & $4(16)$ & $7(33.3)$ & $6(31.6)$ & \\
\hline & Acinetobacter spp. & $0(0)$ & $2(100)$ & $3(100)$ & $0(0)$ & $0(0)$ & \\
\hline & Pseudomonas spp. & $0(0)$ & $6(100)$ & $15(88.2)$ & $45(97.8)$ & $9(100)$ & \\
\hline \multirow[t]{5}{*}{ CRGN } & & 15 (14.9) & $17(16.8)$ & $23(22.8)$ & $42(41.6)$ & $4(4)$ & $<0.0001$ \\
\hline & E. coli & $11(84.6)$ & $11(40.7)$ & $5(13.9)$ & $3(14.3)$ & $0(0)$ & \\
\hline & K. pneumoniae & $2(66.7)$ & $2(40)$ & $0(0)$ & $1(7.1)$ & $0(0)$ & \\
\hline & Acinetobacter spp. & $1(33.3)$ & $3(100)$ & $3(100)$ & $0(0)$ & $0(0)$ & \\
\hline & Pseudomonas spp. & $0(0)$ & $1(16.7)$ & $15(75)$ & $38(82.6)$ & $4(44.4)$ & \\
\hline MRSA & & $0(0)$ & $3(50)$ & $1(25)$ & $0(0)$ & $0(0)$ & 0.038 \\
\hline MR-CONS & & 13 (86.7) & $7(53.8)$ & $13(92.9)$ & 22 (78.6) & $5(62.5)$ & 0.112 \\
\hline
\end{tabular}

MDRGN: multidrug-resistant gram-negative bacteria; ESBL: extended-spectrum beta-lactamases (ESBL)-producing gram-negative bacteria; CRE: carbapenemresistant Enterobacterales; CRGN: carbapenem-resistant gram-negative bacteria; MRCONS: methicillin-resistant coagulase-negative Staphylococci; MRSA: methicillinresistant Staphylococcus aureus. $P$-values marked with bold indicate statistically significant $p$-values

gram-negative BSI ( $p$-value 0.008 and 0.003 , respectively). Furthermore, we found that the odds of MDR gram-negative BSI increased annually during 2016-2018 (Table 3).

Given the emergence of CRGN bacteria isolated in blood culture of patients with BSI during 2015-2018 (as we shown in Fig. 4) and the high proportion of CR isolates in non-fermenters (more than 70\%) and also in Enterobacterales (about 25\%), we investigated the odds of different possible risk factors for carbapenemresistant BSI by logistic regression model (Table 4). Accordingly, we found that age $<60$-year-old, solid organ neoplasms, non-fermenter gram-negative BSI, thirdgeneration cephalosporine resistant gram-negative isolates, and polymyxin-resistance infections were significantly associated with carbapenem-resistant BSI based on univariate logistic regression analysis.

As illustrated in Fig. 7, based on both univariate and multivariate logistic regression analysis, Pseudomonasassociated BSI and polymyxin-resistance BSI were significantly associated with carbapenem-resistant BSI. Among tested antibiotics, only fluoroquinolones (including ciprofloxacin and levofloxacin) and aminoglycosides have acceptable sensitivity (resistance rate lesser than 10\%) for Pseudomonas associated BSI. For E. coli-associated BSIs, polymyxins were the most active drug, while polymyxins and carbapenems were the best choices for $K$. pneumoniae-associated BSI (resistance rate $<15 \%$ ).

PMR: polymyxin-resistant; CR: carbapenem-resistant; 3rdGCR: third-generation cephalosporine-resistant gram-negative isolates; 4thGCR: fourth-generation cephalosporine-resistant gram-negative isolates; AGR: aminoglycoside- resistant; FQR: fluoroquinolone-resistant.

Detail information regarding susceptibility profile of MDR, CR, and ESBL-producer E. coli, Pseudomonas spp., and K. pneumoniae isolates against different antimicrobial classes summarized in Table 5.

\section{Discussion}

Patients with malignancy are predisposed to developing BSI during their chemotherapy courses. Lots of evidence showed that the epidemiology of nosocomial infections in cancer patients changed over the past decades, with the reemergence of GNB as the predominant causative pathogens. The current study, therefore, conducted to describe the antibiotic-resistant patterns and outcomes of nosocomial infections caused by GNB in adult cancer patients.

Overall, despite the annual increase in the admission rate, no significant increase in the BSI incidence rate and attributed death occurred during the five-year study 
Table 2 Univariate logistic regression analysis of variables investigated for prediction of mortality in cancer patients with bloodstream infection

\begin{tabular}{|c|c|c|c|c|}
\hline & Alive $(n=324,78.5 \%)$ & Dead $(n=89,21.5 \%)$ & OR $(95 \% \mathrm{Cl})$ & $p$-value \\
\hline \multicolumn{5}{|l|}{ Sex } \\
\hline Female & $136(45.8)$ & $37(45.7)$ & $1.005(0.613,1.645)$ & 0.986 \\
\hline Male* & $161(54.2)$ & $44(54.3)$ & & \\
\hline \multicolumn{5}{|l|}{ Age } \\
\hline$<60$ & $209(70.4)$ & $52(64.2)$ & $1.325(0.789,2.224)$ & 0.288 \\
\hline$>60^{*}$ & $88(29.6)$ & 29(35.8) & & \\
\hline \multicolumn{5}{|l|}{ Malignancy type } \\
\hline Solid organ tumor* & $131(44.1)$ & $36(44.4)$ & $1.014(0.618,1.662)$ & 0.957 \\
\hline Hematologic malignancy & $166(55.9)$ & $45(55.6)$ & & \\
\hline ESR & $73.58(36.10)$ & $63.77(36.88)$ & $1.008(0.999,1.017)$ & 0.091 \\
\hline CRP & $82.12(43.12)$ & $88.36(28.62)$ & $0.996(0.989,1.004)$ & 0.332 \\
\hline \multicolumn{5}{|l|}{ WBC count } \\
\hline$<4000 /\left.\mu\right|^{*}$ & $169(57.3)$ & $49(60.5)$ & $1.142(0.691,1.885)$ & 0.605 \\
\hline$>4000 / \mu \mathrm{l}$ & $126(42.7)$ & $32(39.5)$ & & \\
\hline \multicolumn{5}{|l|}{ Neutropenia } \\
\hline Yes $(<1500 / \mu \mathrm{l})$ & $121(41.3)$ & $33(41.3)$ & $1.002(0.606,1.656)$ & 0.994 \\
\hline No $(>1500 / \mu l) *$ & $172(58.7)$ & $47(58.8)$ & & \\
\hline \multicolumn{5}{|l|}{ Febrile neutropenia } \\
\hline No & $212(71.4)$ & $60(74.1)$ & $0.873(0.500,1.524)$ & 0.633 \\
\hline Yes* $^{*}$ & $85(28.6)$ & $21(25.9)$ & & \\
\hline \multicolumn{5}{|l|}{ Year } \\
\hline 2015 & $36(12.1)$ & $6(7.4)$ & $1.773(0.612,5.132)$ & 0.291 \\
\hline 2016 & $52(17.5)$ & $11(13.6)$ & $1.397(0.569,3.427)$ & 0.466 \\
\hline 2017 & $81(27.3)$ & $22(27.2)$ & $1.088(0.500,2.368)$ & 0.832 \\
\hline 2018 & $84(28.3)$ & $29(35.8)$ & $0.856(0.405,1.810)$ & 0.684 \\
\hline $2019^{*}$ & $44(14.8)$ & $13(16)$ & & \\
\hline \multicolumn{5}{|l|}{ Enterobacterales } \\
\hline $\mathrm{No}^{*}$ & $70(36.1)$ & $18(38.3)$ & $1.100(0.570,2.121)$ & 0.777 \\
\hline Yes & $124(63.9)$ & $29(61.7)$ & & \\
\hline
\end{tabular}

* Reference

period (Fig. 2). Like some other reports, our study showed that CLABSI was more common in solid tumors than hematologic malignancies [19]; however, a higher prevalence of CLABSI detected in patients with hematological malignancies in other studies [20]. We found that gramnegative BSI was the most common etiology of BSI in cancer patients $(63.3 \%)$, which agrees with other reports [21, 22]. Like recent reports, we observed a gradual increase in the incidence of MDRGN and CRGN associated BSIs annually during our surveillance $[23,24]$.

E. coli, Pseudomonas spp., and K. pneumoniae were the most common recovered gram-negative isolates in our study, which is in line with previous studies conducted in cancer patients [12, 21, 22, 25]. We found a high proportion of ESBL producers and carbapenemresistant isolates, predominantly in non-fermenters (96.4 and $82.3 \%$ ) and the Enterobacteriaceae family (64.1 and $28.8 \%)$. Although there are scarce reports on the incidence of carbapenem-resistant GNs in our region, obtained results are comparable to available reports [26]; however, compared to Europe, North America, Latin America global surveillance studies, and Asia-Pacific regional surveillance studies, our results showed significant higher-resistance rate [27]. We observed that the incidence of MDR BSI increased from 2015 to 2018.

The overall mortality rate of GNB BSIs among cancer patients in our study was about $20 \%$, which was more significant compared with studies conducted in our 
Table 3 Univariate logistic regression analysis of variables investigated for prediction of MDR gram-negative infection in cancer patients with bloodstream infection

\begin{tabular}{|c|c|c|c|c|}
\hline & Non-MDR $(n=19,50.4 \%)$ & MDR $(n=188,49.6 \%)$ & OR $(95 \% \mathrm{Cl})$ & $p$-value \\
\hline \multicolumn{5}{|l|}{ Sex } \\
\hline Female & $78(40.8)$ & $95(50.5)$ & $1.48(0.986,2.221)$ & 0.059 \\
\hline Male* & $113(59.2)$ & $93(49.5)$ & & \\
\hline \multicolumn{5}{|l|}{ Age } \\
\hline$<60$ & $125(65.4)$ & $136(72.3)$ & $1.381(0.892,2.138)$ & 0.148 \\
\hline$>60^{*}$ & $66(34.6)$ & $52(27.7)$ & & \\
\hline \multicolumn{5}{|l|}{ Malignancy } \\
\hline Solid organ tumor & $84(44)$ & $83(44.1)$ & $1.007(0.671,1.511)$ & 0.973 \\
\hline Hematologic malignancy* & $107(56)$ & $105(55.9)$ & & \\
\hline ESR & $67.96(34.76)$ & $75.40(37.68)$ & $1.006(0.999,1.013)$ & 0.101 \\
\hline CRP & $78.60(37.28)$ & $88.05(43.48)$ & $1.006(1.000,1.012)$ & 0.065 \\
\hline \multicolumn{5}{|l|}{ WBC count } \\
\hline$<4000 / \mu l$ & $97(51.3)$ & $122(64.9)$ & $1.753(1.159,2.651)$ & 0.008 \\
\hline$>4000 /\left.\mu\right|^{*}$ & $92(48.7)$ & $66(35.1)$ & & \\
\hline \multicolumn{5}{|l|}{ Neutropenia } \\
\hline Yes $(<1500 / \mu l)$ & $69(36.7)$ & $86(46.2)$ & $1.483(0.981,2.243)$ & 0.062 \\
\hline No $(>1500 / \mu l) *$ & $119(63.3)$ & $100(53.8)$ & & \\
\hline \multicolumn{5}{|l|}{ Febrile neutropenia } \\
\hline $\mathrm{No}^{*}$ & $47(24.6)$ & $59(31.4)$ & $1.401(0.893,2.200)$ & 0.142 \\
\hline Yes & $144(75.4)$ & $129(68.6)$ & & \\
\hline \multicolumn{5}{|l|}{ Year } \\
\hline 2015 & $24(12.6)$ & $18(9.6)$ & $0.960(0.429,2.146)$ & 0.921 \\
\hline 2016 & $32(16.8)$ & $31(16.5)$ & $1.240(0.604,2.546)$ & 0.558 \\
\hline 2017 & $51(26.7)$ & $52(27.7)$ & $1.305(0.681,2.501)$ & 0.422 \\
\hline 2018 & $52(27.2)$ & $62(33)$ & $1.526(0.805,2.894)$ & 0.195 \\
\hline $2019^{*}$ & $32(16.8)$ & $25(13.3)$ & & \\
\hline \multicolumn{5}{|l|}{ Enterobacterales } \\
\hline No ** & $44(81.5)$ & $110(58.8)$ & $3.120(1.481,6.575)$ & 0.003 \\
\hline Yes* & $10(18.5)$ & $78(41.5)$ & & \\
\hline
\end{tabular}

* Reference

** non-fermenter gram-negative BSI. $P$-values marked with bold indicate statistically significant $p$-values

region, for example, Calik Basaran et al. (17.0\%) [28] and Garcia-Vidal et al. (14.8\%) [12]; however, is much lower than some other studies, for example, Al-Otaibi et al. (32.1\%) [5], and Yawei Zhang et al. (33.5\%) [29].

The mortality rate of carbapenem-resistant Enterobacterales, ESBL producing Enterobacterales, MDR Acinetobacter spp., and MDR P. aeruginosa is around $6-7 \%$ in the US, and Europe reports [24, 30]. However, in this study, the BSI attributed mortality rate among MDRGN, CRGN, ESBL producer, Pseudomonas spp., E. coli, and K. pneumoniae were 20.2, 18.8, 19.1, 22, 22.1, and 21.5\%, respectively.
We did not observe an association between investigated factors and survival; however, in a study by Chien-Yuan Chen et al. age $\geq 60$ years, prior allogeneic transplantation and BSI due to VRE found as independent predictors for mortality [31]. CRGN BSI [26, 32-34], unresolved neutropenia, monotherapy, septic shock [32, 35], and polymicrobial BSI [32] are other risk factors associated with increased mortality in cancer patients with GN BSI.

We identified MDR gram-negative infections more likely to occur in patients with WBC count $<4000$ and non-fermenter gram-negative BSI (Table 3). Other studies found that male sex, age $\geq 60$, previous antimicrobial 
Table 4 Logistic regression analysis of factors associated with carbapenem-resistant gram-negative bacterial bloodstream infection in cancer patients

\begin{tabular}{|c|c|c|c|c|}
\hline & n (\%) & n (\%) & Univariate & $p$-value \\
\hline \multicolumn{5}{|l|}{ Age } \\
\hline$<60$ & $78(83)$ & $54(56.8)$ & $3.701(1.887,7.262)$ & $<0.0001$ \\
\hline$>60^{*}$ & $16(17)$ & $41(43.2)$ & & \\
\hline \multicolumn{5}{|l|}{ Malignancy type } \\
\hline Solid organ & $55(58.5)$ & $35(36.8)$ & $2.418(1.347,4.339)$ & 0.003 \\
\hline Hematologic malignancy* & $39(41.5)$ & $60(63.2)$ & & \\
\hline \multicolumn{5}{|l|}{ Enterobacterales } \\
\hline Yes* $^{*}$ & $33(35.1)$ & $81(85.3)$ & $10.695(5.269,21.709)$ & $<0.0001$ \\
\hline No & $61(64.9)$ & $14(14.7)$ & & \\
\hline \multicolumn{5}{|l|}{ 4th GCRGN } \\
\hline Resistance & $42(44.7)$ & $31(32.6)$ & $1.667(0.923,3.011)$ & 0.090 \\
\hline Sensitive* & $52(55.3)$ & $64(67.4)$ & & \\
\hline \multicolumn{5}{|l|}{ 3rd GCRGN } \\
\hline Resistance & 88 (93.6) & $57(60)$ & $9.778(3.884,24.615)$ & $<0.0001$ \\
\hline Sensitive* & $6(6.4)$ & $38(40)$ & & \\
\hline \multicolumn{5}{|l|}{ Aminoglycoside-resistant } \\
\hline Resistance & $31(33)$ & $21(22.1)$ & $1.734(0.907,3.314)$ & 0.096 \\
\hline Sensitive* & $63(67)$ & $74(77.9)$ & & \\
\hline \multicolumn{5}{|l|}{ Polymyxin-resistance } \\
\hline Resistance & $50(53.2)$ & $6(6.3)$ & $16.856(6.713,42.323)$ & $<0.0001$ \\
\hline Sensitive* & $44(46.8)$ & 89 (93.7) & & \\
\hline
\end{tabular}

* Reference. $P$-values marked with bold indicate statistically significant $p$-values

use, liver disease, and bacteremia caused by $K$. pneumoniae are associated with increased risk of MDRGN BSI [36].

In agreement with current concerns regarding the efficacy of colistin against carbapenem-resistant pathogens, including CRE [37-40], we found that CR E. coli and $K$. pneumoniae isolates did not show acceptable sensitivity to our available antimicrobial choices, including colistin. However, ESBL producer $K$. pneumoniae and ESBL E. coli isolates had acceptable sensitivity to carbapenems and aminoglycosides (>80\%) and polymyxins (84.3\%), respectively. Like the previous reports, our study reemphasized that carbapenems could still act as the drugs of choice in ESBL associated BSIs in cancer patients [41]. Besides, MDR E. coli and $K$. pneumoniae showed a high resistance rate to available antimicrobial agents. Our findings are consistent with previous studies concerning the global emergence of MDRGN pathogens as a significant healthcare burden that could be attributed to the overuse of antibiotics and necessitates the development of new antibiotics for treating CRE, CR P. aeruginosa, and CR Acinetobacter baumannii [42-44].
We also found that MDR, CR, and ESBL Pseudomonas spp. isolates still are sensitive to ciprofloxacin in our setting and could be considered a good treatment choice as recommended by current guidelines [45]. Remarkably, piperacillin-tazobactam, which is frequently preferred as one of the initial antibiotic therapies in our febrile neutropenic patients, may not be an excellent empiric choice against CR E. coli and K. pneumoniae. Our study showed that carbapenems might be less active against ESBL-producer Pseudomonas spp. and to some extent against ESBLproducer E. coli. In MDRGN BSI, none of our empiric treatments (carbapenems and piperacillintazobactam) could overcome GN bacteremia and need other choices such as colistin (Table 5). Although febrile neutropenia should be considered a medical emergency and a prompt administration of empirical antibiotic therapy is mandatory, increased mortality could be seen due to inappropriate empiric antibiotic therapy in setting with high rates of resistant pathogens $[2,12,19,46]$. Accordingly, regular epidemiological and microbiological surveillance of BSI should be encouraged strongly in oncology centers. 

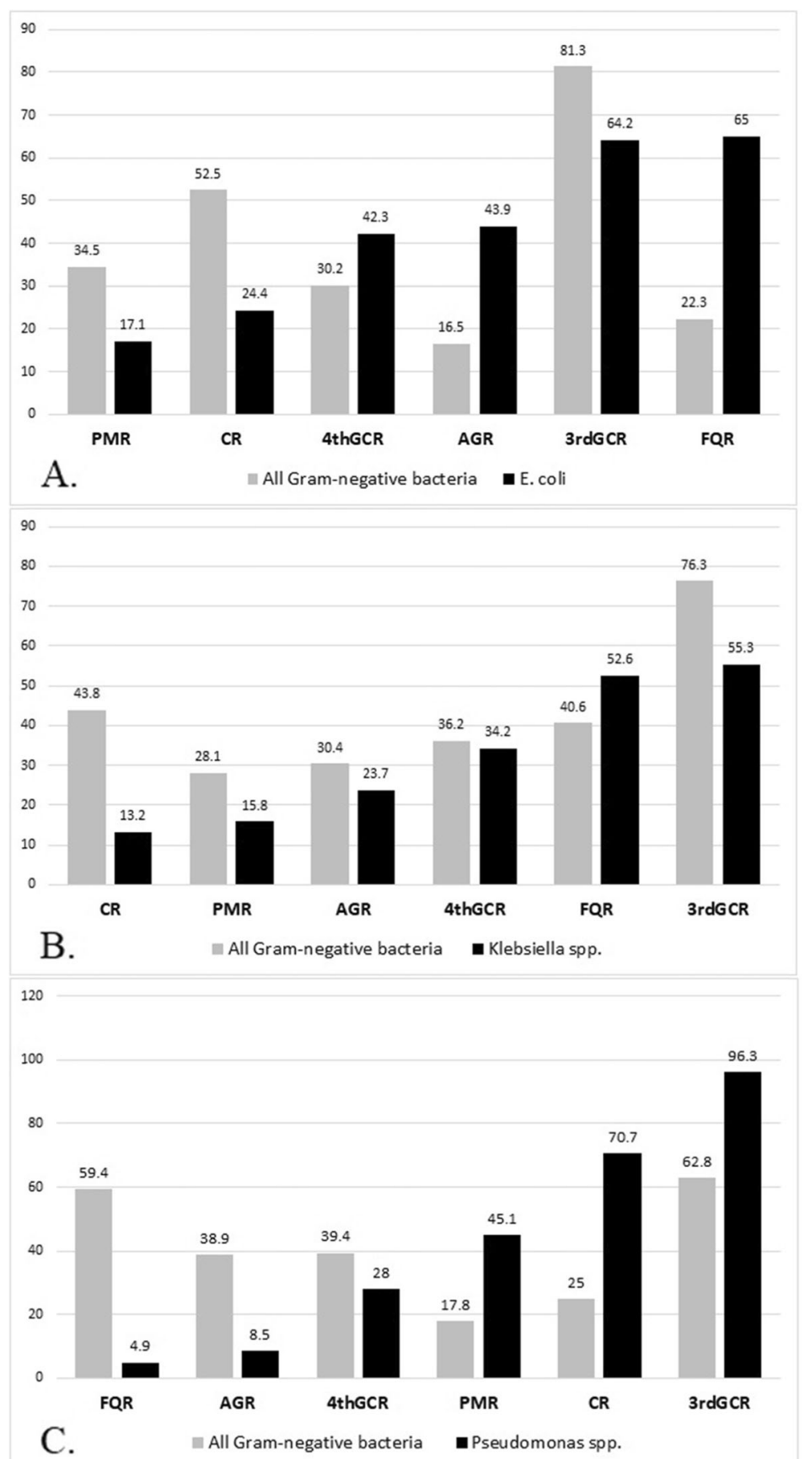

Fig. 7 Comparative analysis for antibiotic resistance rate between E. coli, K. pneumoniae, and Pseudomonas spp. with all gram-negative isolates (A, $B$, and $C$, respectively)

\section{Limitation}

Our study has some limitations. First, given the retrospective nature of this study, it was difficult to collect some variables (chemotherapeutic protocols, antibiotics treatment before admission, and some clinical and laboratory examination results) in this retrospective study.
So, there might be hidden biases in the analysis of the relationship. Second, carbapenemase type and enzyme were not investigated in this study. Finally, our research was conducted with data collected from a single center. Therefore, a prospective multicenter study is needed for further validation. 
Table 5 The susceptibility profile of carbapenem-resistant and ESBL-producer E. coli, Pseudomonas spp., and K. pneumoniae isolates against different antimicrobial classes

\begin{tabular}{|c|c|c|c|c|c|c|}
\hline Sensitivity rate $(n, \%)$ & 4th GC & Ciprofloxacin & AGs & BL/BLI & Polymyxins & Carbapenems \\
\hline \multicolumn{7}{|l|}{ Carbapenem-resistant } \\
\hline E. coli & $16(53.3 \%)$ & $8(26.7 \%)$ & $9(30 \%)$ & 11 (36.7\%) & $18(69.2 \%)$ & - \\
\hline Pseudomonas spp. & $35(60.3 \%)$ & $54(93.1 \%)$ & $51(78.9 \%)$ & 47 (81\%) & $6(66.6 \%)$ & - \\
\hline K. pneumoniae & $2(40 \%)$ & $1(20 \%)$ & $2(25 \%)$ & $2(40 \%)$ & $5(62.5 \%)$ & - \\
\hline \multicolumn{7}{|l|}{ ESBL } \\
\hline E. coli & $20(28.6 \%)$ & $17(24.3 \%)$ & $38(54.3 \%)$ & $21(30 \%)$ & 59 (84.3\%) & $51(72.9 \%)$ \\
\hline Pseudomonas spp. & $52(69.3 \%)$ & 71 (94.7\%) & 70 (93.3\%) & $53(70.7 \%)$ & 41 (54.7\%) & $20(26.7 \%)$ \\
\hline K. pneumoniae & $7(35 \%)$ & $6(30 \%)$ & $16(80 \%)$ & $1(5 \%)$ & $6(75 \%)$ & 17 (85\%) \\
\hline \multicolumn{7}{|l|}{ MDR } \\
\hline E. coli & $42(46.2 \%)$ & 19 (20.9\%) & 40 (44\%) & $27(29.7 \%)$ & 71 (78\%) & $62(68.1 \%)$ \\
\hline Pseudomonas spp. & $51(68.9 \%)$ & 70 (94.6\%) & 67 (90.5\%) & 49 (66.2\%) & 37 (50\%) & $18(24.3 \%)$ \\
\hline K. pneumoniae & 11 (45.8\%) & $6(25 \%)$ & 15 (62.5\%) & $3(12.5 \%)$ & 19 (79.2\%) & 19 (79.2\%) \\
\hline
\end{tabular}

$4^{\text {th }}$ GC: fourth-generation cephalosporins (cefepime); AGs: aminoglycosides (amikacin and gentamicin); BL/BLI: beta-lactamase/beta-lactamase inhibitors combination (piperacillin-tazobactam)

\section{Conclusions}

In conclusion, the overall case-fatality rate of BSI caused by GNB among cancer patients was $20 \%$. The high proportion of the most frequently isolated pathogens were $\mathrm{CR}$ and ESBL-producing Enterobacterales and Pseudomonas spp. Although fluoroquinolones remain a good choice for CR $P$. aeruginosa, we have few effective choices against CR E. coli and K. pneumoniae. The new agents such as ceftazidime/avibactam could be helpful in such cases.

\section{Abbreviations}

BSI: bloodstream infections; CLABSI: Central Line-Associated BSI;

ASP: Antimicrobial stewardship program; GN: gram-negative;

CR: Carbapenem-resistant; MRCoNS: Methicillin-resistant coagulase-negative staphylococci; ESBL: extended-spectrum beta-lactamase; CRE: Carbapenemresistant Enterobacterales; CRGN: Carbapenem-resistant gram-negative; MDRG $\mathrm{N}$ : multi-drug resistance gram-negative

\section{Supplementary Information}

The online version contains supplementary material available at https://doi. org/10.1186/s12879-021-06243-z.

Additional file 1: Table S1. The summary characteristics of the patients with solid tumors and hematological malignancies.

\section{Acknowledgments}

Our thanks go to Infection Control Unit staffs in Amir Medical Oncology Hospital, Shiraz University of Medical Sciences, for their technical support and assistance.

\section{Authors' contributions}

Study concept and design: AA; Acquisition of data: GS, KS, SZ, and MS; Statistical Analysis: AA and SZ, Analysis and interpretation of data: AA and NS; Drafting of the manuscript: AA, SS, and SZ, Critical revision of the manuscript for important intellectual content: AA; Study supervision: AA and SZ. All individuals listed as (co)-authors have met the authorship criteria, and nobody who qualifies for authorship omitted from the list. The final manuscript was corrected and approved by all authors.

\section{Funding}

Not applicable.

Availability of data and materials

The datasets used and analyzed during the current study are available from the corresponding author on reasonable request.

\section{Declarations}

\section{Ethics and consent to participate}

The project was found to be in accordance with the ethical principles and the national norms and standards for conducting Medical Research in Iran with approval ID IR.SUMS.MED.REC.1399.082 on 2020-05-18 [47].

\section{Consent for publication}

Not applicable.

\section{Competing interests}

The authors do not have any financial or other relationships, which could regard as a conflict of interest.

\section{Author details}

${ }^{1}$ Professor Alborzi Clinical Microbiology Research Center, Shiraz University of Medical Sciences, Shiraz, Iran. ${ }^{2}$ Shiraz University of Medical Sciences, Shiraz 7193711351, Iran.

Received: 27 November 2020 Accepted: 26 May 2021

Published online: 02 July 2021

\section{References}

1. El-Mahallawy HA, Hassan SS, El-Wakil M, Moneer MM, Shalaby L. Increasing antimicrobial resistance monitored in surveillance analysis of blood stream infections in febrile neutropenic pediatric oncology patients. Asian Pac J Cancer Prev. 2015;16(14):5691-5. https://doi.org/10.7314/APJCP.2015.16.14. 5691

2. Kang C-I, Kim S-H, Park WB, Lee K-D, Kim H-B, Kim E-C. Oh M-d, Choe K-W: bloodstream infections caused by antibiotic-resistant gram-negative bacilli: risk factors for mortality and impact of inappropriate initial antimicrobial therapy on outcome. Antimicrob Agents Chemother. 2005;49(2):760-6. https://doi.org/10.1128/AAC.49.2.760-766.2005.

3. Van de Louw A, Lewis AM, Yang Z. Autopsy findings in patients with acute myeloid leukemia and non-Hodgkin lymphoma in the modern era: a focus on lung pathology and acute respiratory failure. Ann Hematol. 2019;98(1): 119-29. https://doi.org/10.1007/s00277-018-3494-3. 
4. Easow JM, Joseph NM, Dhungel BA, Chapagain B, Shivananda PG. Blood stream infections among febrile patients attending a teaching hospital in Western region of Nepal. Australasian Medical Journal. 2010;3(10):633-7.

5. Al-Otaibi FE, Bukhari EE, Badr M, Alrabiaa AA. Prevalence and risk factors of gram-negative bacilli causing blood stream infection in patients with malignancy. Saudi Medical Journal. 2016;37(9):979-84. https://doi.org/10.1 5537/smj.2016.9.14211

6. Kokkayil P, Agarwal R, Mohapatra S, Bakhshi S, Das B, Sood S, et al. Bacterial profile and antibiogram of blood stream infections in febrile neutropenic patients with haematological malignancies. Journal of Infection in Developing Countries. 2018;12(6):442-7. https://doi.org/10.3855/jidc.9725.

7. Prabhash K, Medhekar A, Ghadyalpatil N, Noronha V, Biswas S, Kurkure P, et al. Blood stream infections in cancer patients: A single center experience of isolates and sensitivity pattern. Indian J Cancer. 2010;47(2):184-8. https:// doi.org/10.4103/0019-509X.63019.

8. McNamara JF, Righi E, Wright H, Hartel GF, Harris PNA, Paterson DL. Longterm morbidity and mortality following bloodstream infection: A systematic literature review. J Inf Secur. 2018;77(1):1-8.

9. Kuderer NM, Dale DC, Crawford J, Cosler LE, Lyman GH. Mortality, morbidity, and cost associated with febrile neutropenia in adult cancer patients. Cancer. 2006;106(10):2258-66. https://doi.org/10.1002/cncr.21847.

10. Feld R. Bloodstream infections in cancer patients with febrile neutropenia. Int J Antimicrob Agents. 2008;32:S30-3. https://doi.org/10.1016/j.ijantimica g.2008.06.017.

11. Tang $Y$, Wu X, Cheng Q, Li X. Inappropriate initial antimicrobial therapy for hematological malignancies patients with gram-negative bloodstream infections. Infection. 2020;48(1):109-16. https://doi.org/10.1007/s15010-01901370-x.

12. Garcia-Vidal C, Cardozo-Espinola C, Puerta-Alcalde P, Marco F, Tellez A, Agüero $D$, et al. Risk factors for mortality in patients with acute leukemia and bloodstream infections in the era of multiresistance. PLoS One. 2018; 13(6):e0199531. https://doi.org/10.1371/journal.pone.0199531.

13. Doron S, Davidson LE. Antimicrobial stewardship. Mayo Clin Proc. 2011; 86(11):1113-23. https://doi.org/10.4065/mcp.2011.0358.

14. Diekema DJ, Hsueh P-R, Mendes RE, Pfaller MA, Rolston KV, Sader HS, et al. The microbiology of bloodstream infection: 20-year trends from the SENTRY antimicrobial surveillance program. Antimicrob Agents Chemother. 2019; 63(7):e00355-19.

15. Organization $\mathrm{WH}$ : Antimicrobial resistance - global report on surveillance. https://apps.who.int/iris/bitstream/handle/10665/112642/97892415 64748 eng.pdf;jsessionid=519244EEOEEF520027CE4098504150B4? sequence $=1$. 2014.

16. Clinical and Laboratory Standards Institute (CLSI): Performance Standards for Antimicrobial Susceptibility Testing. 30th ed. CLSI supplement M100. In. 950 West Valley Road. Suite 2500, Wayne, Pennsylvania 19087 USA: Clinical and Laboratory Standards Institute; 2020: 108.

17. Clinical and Laboratory Standards Institute (CLSI): Performance standards for antimicrobial susceptibility testing: approved 28th ed. In.: CLSI Wayne, PA; 2018.

18. Magiorakos A-P, Srinivasan A, Carey R, Carmeli Y, Falagas M, Giske C, et al. Multidrug-resistant, extensively drug-resistant and pandrug-resistant bacteria: an international expert proposal for interim standard definitions for acquired resistance. Clin Microbiol Infect. 2012;18(3):268-81. https://doi. org/10.1111/j.1469-0691.2011.03570.x.

19. Islas-Muñoz B, Volkow-Fernández P, Ibanes-Gutiérrez C, Villamar-Ramírez A, Vilar-Compte D, Cornejo-Juárez P. Bloodstream infections in cancer patients. Risk factors associated with mortality. Int J Infect Dis. 2018;71:59-64. https:// doi.org/10.1016/j.ijid.2018.03.022.

20. Moell J, Svenningsson A, Af Sandeberg M, Larsson M, Heyman M, HarilaSaari $A$, et al. Early central line-associated blood stream infections in children with cancer pose a risk for premature catheter removal. Acta Paediatr. 2019;108(2):361-6. https://doi.org/10.1111/apa.14432.

21. Vahedian-Ardakani HA, Moghimi M, Shayestehpour M, Doosti M, Amid N. Bacterial Spectrum and antimicrobial resistance pattern in Cancer patients with febrile neutropenia. Asian Pac J Cancer Prev. 2019;20(5):1471-4. https:// doi.org/10.31557/APJCP.2019.20.5.1471.

22. Jamal A, Fatima N, Shaikh S, Kaleem B, Rizvi QA, Zaidi U, et al. Pattern of antimicrobial sensitivity in microbiologically documented infections in neutropenic patients with Haematological malignancies: A single center study. Indian J Microbiol. 2019;59(2):188-92. https://doi.org/10.1007/s12088019-00789-y.
23. Litwin A, Fedorowicz O, Duszynska W. Characteristics of microbial factors of healthcare-associated infections including multidrug-resistant pathogens and antibiotic consumption at the university intensive care unit in Poland in the years 2011-2018. Int J Environ Res Public Health. 2020;17(19):6943. https://doi.org/10.3390/ijerph17196943.

24. Annual Report of the European Antimicrobial Resistance Surveillance Network (EARS-Net) [https://ecdc.europa.eu/sites/porta/files/documents/EA RS-Net-report-2017-update-jan-2019.pdf.]

25. Andersen MA, Moser CE, Lundgren J, Niemann CU. Epidemiology of bloodstream infections in patients with chronic lymphocytic leukemia: a longitudinal nation-wide cohort study. Leukemia. 2019;33(3):662-70. https:// doi.org/10.1038/s41375-018-0316-5.

26. Righi E, Peri AM, Harris PN, Wailan AM, Liborio M, Lane SW, et al. Global prevalence of carbapenem resistance in neutropenic patients and association with mortality and carbapenem use: systematic review and meta-analysis. J Antimicrob Chemother. 2017;72(3):668-77. https://doi.org/1 0.1093/jac/dkw459.

27. Nordmann P, Poirel L: Epidemiology and diagnostics of carbapenem resistance in gram-negative bacteria. Clinical Infectious Diseases 2019, 69(Supplement_7):S521-S528.

28. Başaran NÇ, Karaağaoğlu E, Hasçelik G, Tanrı̈ver MD, Akova M. Prospective evaluation of infection episodes in cancer patients in a tertiary care academic center: microbiological features and risk factors for mortality. Turkish Journal of Hematology. 2016;33(4):311-9. https://doi.org/10.4274/ tjh.2015.0216.

29. Zhang Y, Wang Q, Yin Y, Chen H, Jin L, Gu B, Xie L, Yang C, Ma X, Li H: Epidemiology of carbapenem-resistant Enterobacteriaceae infections: report from the China CRE Network. Antimicrob Agents Chemother 2018, 62(2).

30. Mehrad B, Clark NM, Zhanel GG, Lynch JP III. Antimicrobial resistance in hospital-acquired gram-negative bacterial infections. Chest. 2015;147(5): 1413-21. https://doi.org/10.1378/chest.14-2171.

31. Chen C-Y, Tien F-M, Sheng W-H, Huang S-Y, Yao M, Tang J-L, et al. Clinical and microbiological characteristics of bloodstream infections among patients with haematological malignancies with and without neutropenia at a medical Centre in northern Taiwan, 2008-2013. Int J Antimicrob Agents. 2017;49(3):272-81. https://doi.org/10.1016/j.jiantimicag.2016.11.009.

32. Andria N, Henig O, Kotler O, Domchenko A, Oren I, Zuckerman T, et al. Mortality burden related to infection with carbapenem-resistant gramnegative bacteria among haematological cancer patients: a retrospective cohort study. J Antimicrob Chemother. 2015;70(11):3146-53. https://doi. org/10.1093/jac/dkv218.

33. Moghnieh R, Estaitieh N, Mugharbil A, Jisr T, Abdallah Dl, Ziade F, et al. Third generation cephalosporin resistant Enterobacteriaceae and multi-drug resistant gram-negative bacteria causing bacteremia in febrile neutropenia adult cancer patients in Lebanon, broad spectrum antibiotics use as a major risk factor, and correlation with poor prognosis. Front Cell Infect Microbiol. 2015;5:11.

34. Kim Y-a, Yong choi J, Ki kim C, Oh kim C, Soo kim M, Hoon choi S, Sik chin $\mathrm{B}$, Hoon han S, Sung lee $\mathrm{H}$, Kyoung choi $\mathrm{H}$ : Risk factors and outcomes of bloodstream infections with metallo- $\beta$-lactamase-producing Acinetobacter. Scand J Infect Dis 2008, 40(3):234-240.

35. Tofas P, Skiada A, Angelopoulou M, Sipsas N, Pavlopoulou I, Tsaousi S, et al. Carbapenemase-producing Klebsiella pneumoniae bloodstream infections in neutropenic patients with haematological malignancies or aplastic anaemia: analysis of 50 cases. Int J Antimicrob Agents. 2016;47(4):335-9. https://doi. org/10.1016/j.jantimicag.2016.01.011.

36. Leal HF, Azevedo J, Silva GEO, Amorim AML, de Roma LRC, Arraes ACP, et al. Bloodstream infections caused by multidrug-resistant gram-negative bacteria: epidemiological, clinical and microbiological features. BMC Infect Dis. 2019;19(1):609. https://doi.org/10.1186/s12879-019-4265-z.

37. Mansour W, Haenni M, Saras E, Grami R, Mani Y, Khalifa ABH, et al. Outbreak of colistin-resistant carbapenemase-producing Klebsiella pneumoniae in Tunisia. Journal of global antimicrobial resistance. 2017;10:88-94. https://doi. org/10.1016/j.jgar.2017.03.017.

38. El-Mokhtar MA, Daef E, Mohamed Hussein AA, Hashem MK, Hassan HM. Emergence of nosocomial pneumonia caused by Colistin-resistant Escherichia coli in patients admitted to chest intensive care unit. Antibiotics. 2021;10(3):226. https://doi.org/10.3390/antibiotics10030226.

39. Paterson DL, Harris PN. Colistin resistance: a major breach in our last line of defence. Lancet Infect Dis. 2016;16(2):132-3. https://doi.org/10.1016/S1473-3 099(15)00463-6. 
40. Hussein NH, Al-Kadmy IM, Taha BM, Hussein JD. Mobilized colistin resistance (mcr) genes from 1 to 10: a comprehensive review. Mol Biol Rep. 2021:1-11.

41. Rodríguez-Baño J, Gutiérrez-Gutiérrez B, Machuca I. Pascual A: Treatment of infections caused by extended-spectrum-beta-lactamase-, AmpC-, and carbapenemase-producing Enterobacteriaceae. Clin Microbiol Rev. 2018: $31(2)$.

42. Shrivastava SR, Shrivastava PS, Ramasamy J. World health organization releases global priority list of antibiotic-resistant bacteria to guide research, discovery, and development of new antibiotics. Journal of Medical Society. 2018;32(1):76. https://doi.org/10.4103/jms.jms_25_17.

43. Abadi ATB, Rizvanov AA, Haertlé T, Blatt NL. World Health Organization report: current crisis of antibiotic resistance. BioNanoScience. 2019;9(4):77888. https://doi.org/10.1007/s12668-019-00658-4.

44. Zhou C, Jin L, Wang Q, Wang X, Chen F, Gao Y, et al. Bloodstream infections caused by Carbapenem-resistant Enterobacterales: risk factors for mortality, antimicrobial therapy and treatment outcomes from a prospective multicenter study. Infection and Drug Resistance. 2021;14:731.

45. Tamma PD, Aitken SL, Bonomo RA, Mathers AJ, van Duin D, Clancy CJ. Infectious Diseases Society of America guidance on the treatment of extended-Spectrum $\beta$-lactamase producing Enterobacterales (ESBL-E), Carbapenem-resistant Enterobacterales (CRE), and Pseudomonas aeruginosa with difficult-to-treat resistance (DTR-P. aeruginosa). Clin Infect Dis. 2021; 72(7):e169-83. https://doi.org/10.1093/cid/ciaa1478.

46. Lodise TP Jr, PN, Kwa A, Graves J, Furuno JP, Graffunder E, Lomaestro B, McGregor JC. Predictors of 30-day mortality among patients with Pseudomonas aeruginosa bloodstream infections: impact of delayed appropriate antibiotic selection. Antimicrob Agents Chemother. 2007;51(10): 3510-5. https://doi.org/10.1128/AAC.00338-07.

47. Research Ethics Certificate [https://ethics.research.ac.ir/ProposalCertificateEn. php?id=133949\&Print=true\&NoPrintHeader=true\&NoPrintFooter $=$ true\&NoPrintPageBorder=true\&LetterPrint=true].

\section{Publisher's Note}

Springer Nature remains neutral with regard to jurisdictional claims in published maps and institutional affiliations.

Ready to submit your research? Choose BMC and benefit from:

- fast, convenient online submission

- thorough peer review by experienced researchers in your field

- rapid publication on acceptance

- support for research data, including large and complex data types

- gold Open Access which fosters wider collaboration and increased citations

- maximum visibility for your research: over $100 \mathrm{M}$ website views per year

At $\mathrm{BMC}$, research is always in progress.

Learn more biomedcentral.com/submissions 